\title{
ON THE NUMBER OF EIGENVALUES OF A MODEL OPERATOR ASSOCIATED TO A SYSTEM OF THREE-PARTICLES ON LATTICES
}

\author{
SERGIO ALBEVERIO ${ }^{1,2,3}$, SAIDAKHMAT N. LAKAEV $^{4,5}$, ZAHRIDDIN I. MUMINOV $^{5}$
}

\begin{abstract}
A model operator $H$ associated to a system of three-particles on the three dimensional lattice $\mathbb{Z}^{3}$ and interacting via pair non-local potentials is studied. The following results are proven: (i) the operator $H$ has infinitely many eigenvalues lying below the bottom of the essential spectrum and accumulating at this point, in the case, where both Friedrichs model operators $h_{\mu_{\alpha}}(0), \alpha=1,2$, have threshold resonances. (ii) the operator $H$ has a finite number of eigenvalues lying outside of the essential spectrum, in the case, where at least one of $h_{\mu_{\alpha}}(0), \alpha=1,2$, has a threshold eigenvalue.
\end{abstract}

Subject Classification: Primary: 81Q10, Secondary: 35P20, 47N50

Key words and phrases: Friedrichs model, pair non-local potentials, infinitely many eigenvalues, Efimov effect, Hilbert-Schmidt operators, conditionally negative definite functions.

\section{INTRODUCTION}

The main goal of the present paper is to prove the finiteness or infiniteness of the number of eigenvalues for a model operator $H$ with emphasis on the asymptotics for the number of infinitely many eigenvalues (Efimov's effect case). The model operator $H$ is associated to a system of three-particles on the lattice $\mathbb{Z}^{3}$ interacting via pair non-local potentials.

The Efimov effect is one of the most remarkable results in the spectral analysis for continuous three-particle Schrödinger operators: if none of the three two-particle Schrödinger operators (corresponding to the two-particle subsystems) has negative eigenvalues, but at least two of them have a zero energy resonance, then this three-particle Schrödinger operator has an infinite number of discrete eigenvalues, accumulating at zero.

Since its discovery by Efimov in $[\overline{8} \mid$ many works have been devoted to this subject. See, for example [1, 6, 7, 13, 19, 22, 23, 24, 25].

The main result obtained by Sobolev [22] (see also [24]) is an asymptotics of the form $\mathcal{U}_{0}|\log | \lambda||$ for the number $N(\lambda)$ of eigenvalues on the left of $\lambda, \lambda<0$, where the coefficient $\mathcal{U}_{0}$ does not depend on the two-particle potentials $v_{\alpha}$ and is a positive function of the ratios $m_{1} / m_{2}, m_{2} / m_{3}$ of the masses of the three-particles.

In models of solid state physics [9, 10, 17, 18, 20, 27| and also in lattice quantum field theory [16] discrete Schrödinger operators are considered, which are lattice analogues of the three-particle Schrödinger operator in a continuous space. The presence of the Efimov effect for these operators was proved in [3, 14 15].

In [3] a system of three arbitrary quantum particles on the lattice $\mathbb{Z}^{3}$ interacting via zero-range pair attractive (local) potentials is considered and for the number of eigenvalues $N(\lambda)$ an asymptotics analogous to [22, 24] have been obtained.

In all papers devoted to Efimov's effect systems of three particles interacting via pair local potentials have been considered.

Date: November 20, 2018. 
In the present paper we study the model operator $H$ associated to a system of threeparticles on $\mathbb{Z}^{3}$ acting in the Hilbert space $L_{2}\left(\left(\mathbb{T}^{3}\right)^{2}\right)$ and interacting via pair non-local potentials, where the role of the two-particle discrete Schrödinger operators is played by a family of Friedrichs models with parameters $h_{\mu_{\alpha}}(p), \alpha=1,2, p \in \mathbb{T}^{3}$.

Under some natural conditions on the family of the operators $h_{\mu_{\alpha}}(p), \alpha=1,2, p \in \mathbb{T}^{3}$, we obtain the following results:

(i) The essential spectrum of it is described via the spectr of the Friedrichs models $h_{\mu_{\alpha}}(p), \alpha=1,2, p \in(-\pi, \pi]^{3}$.

(ii) the operator $H$ has infinitely many eigenvalues lying below the bottom and accumulating at the bottom of its essential spectrum, in the case, where both operators $h_{\mu_{\alpha}}(0), \alpha=1,2$, have threshold energy resonances. Moreover for the number $N(z)$ of eigenvalues of $H$ lying below $z<m=\inf \sigma_{\text {ess }}(H)$ the following limit exists

$$
\lim _{z \rightarrow m-0} \frac{N(z)}{|\log | m-z||}=\mathcal{U}_{0} \quad\left(0<\mathcal{U}_{0}<\infty\right) .
$$

(iii) the operator $H$ has a finite number of eigenvalues lying below the bottom of its essential spectrum in the case, where at least one of the Friedrichs models $h_{\mu_{\alpha}}(0), \alpha=1,2$, has a threshold eigenvalue.

We remark that the assertion (ii) is similar to the case of the three-particle continuous and discrete Schrödinger operators and the assertion (iii) is surprising. Similar assertions do not seem to have been yet proved for the three-particle Schrödinger operators on $\mathbb{R}^{3}$ and $\mathbb{Z}^{3}$.

The plan of this paper is as follows:

Section 1 is an introduction to the whole work. In Section 2 the model operator $H$ is introduced and the main results of the present paper are formulated. In Section 3 we recall concepts and results concerning the threshold analysis of the family of Friedrichs models. In section 4 we study the location of the essential spectrum and prove a realization of the Birman-Schwinger principle for $H$. The finiteness of the number of eigenvalues of the operator $H$ is proved in Section 5. In Section 6 an asymptotic formula for the number of eigenvalues of $H$ is obtained.

Throughout the present paper we adopt the following conventions: The subscript $\alpha$ (and also $\beta$ ) always is equal to 1 or 2 and $\alpha \neq \beta$ and $\mathbb{T}^{3}$ denotes the three-dimensional torus, the cube $(-\pi, \pi]^{3}$ with appropriately identified sides. For each $\delta>0$ the notation $U_{\delta}(0)=\left\{p \in \mathbb{T}^{3}:|p|<\delta\right\}$ stands for a $\delta$-neighborhood of the origin.

Denote by $L_{2}(\Omega)$ the Hilbert space of square-integrable functions defined on a measurable set $\Omega \subset \mathbb{R}^{n}$, and by $L_{2}^{(2)}(\Omega)$ the Hilbert space of two-component vector functions $f=\left(f_{1}, f_{2}\right), f_{\alpha} \in L_{2}(\Omega), \alpha=1,2$. We denote by $\operatorname{diag}\left\{B_{1}, B_{2}\right\}$ the $2 \times 2$ diagonal matrix with operators $B_{1}, B_{2}$ as diagonal entries.

Let $\mathcal{B}\left(\theta, \mathbb{T}^{3}\right)$ with $1 / 2<\theta \leq 1$, be the Banach spaces of Hölder continuous functions on $\mathbb{T}^{3}$ with exponent $\theta$ obtained by the closure of the space of smooth (periodic) functions $f$ on $\mathbb{T}^{3}$ with respect to the norm

$$
\|f\|_{\theta}=\sup _{\substack{t, \ell \in \mathbb{T}^{3} \\ \ell \neq 0}}\left[|f(t)|+|\ell|^{-\theta}|f(t+\ell)-f(t)|\right] .
$$

The set of functions $f: \mathbb{T}^{3} \rightarrow \mathbb{R}$ having continuous partial derivatives up to order $n$ inclusive will be denoted $C^{(n)}\left(\mathbb{T}^{3}\right)$. In particular $C^{(0)}\left(\mathbb{T}^{3}\right)=C\left(\mathbb{T}^{3}\right)$ by our convention that $f^{(0)}(x)=f(x)$. 


\section{THREE PARTICLE MODEL OPERATOR AND STATEMENT OF THE RESULTS}

Let us consider the operator $H$ acting in the Hilbert space $L_{2}\left(\left(\mathbb{T}^{3}\right)^{2}\right)$ by

$$
H=H_{0}-\mu_{1} V_{1}-\mu_{2} V_{2},
$$

where

$$
\left(H_{0} f\right)(p, q)=u(p, q) f(p, q), \quad f \in L_{2}\left(\left(\mathbb{T}^{3}\right)^{2}\right)
$$

and $V_{\alpha}, \alpha=1,2$, are non-local interaction operators

$$
\begin{aligned}
& \left(V_{1} f\right)(p, q)=\varphi_{1}(p) \int_{\mathbb{T}^{3}} \varphi_{1}(t) f(t, q) d t, \quad f \in L_{2}\left(\left(\mathbb{T}^{3}\right)^{2}\right), \\
& \left(V_{2} f\right)(p, q)=\varphi_{2}(q) \int_{\mathbb{T}^{3}} \varphi_{2}(t) f(p, t) d t, \quad f \in L_{2}\left(\left(\mathbb{T}^{3}\right)^{2}\right) .
\end{aligned}
$$

Here $u$ is a real-valued essentially bounded function on $\left(\mathbb{T}^{3}\right)^{2}$ and $\varphi_{\alpha}, \alpha=1,2$, are real-valued functions and belong to $L_{2}\left(\mathbb{T}^{3}\right)$ and $\mu_{\alpha}, \alpha=1,2$, are positive real numbers.

Under these assumptions the operator $H$ defined by 2.1 is bounded and self-adjoint.

Throughout this paper we assume the following additional hypotheses.

Hypothesis 2.1. ( $i$ ) The function $u$ is even on $\left(\mathbb{T}^{3}\right)^{2}$ with respect to $(p, q)$, and has a unique non-degenerate minimum at the point $(0,0) \in\left(\mathbb{T}^{3}\right)^{2}$ and all third order partial derivatives of $u$ belong to $\mathcal{B}\left(\theta,\left(\mathbb{T}^{3}\right)^{2}\right), \frac{1}{2}<\theta \leq 1$.

(ii) For some positive definite matrix $U$ and real numbers $l, l_{1}, l_{2}\left(l_{1}, l_{2}>0, l \neq 0\right)$ the following equalities hold

$$
\left(\frac{\partial^{2} u(0,0)}{\partial p^{(i)} \partial p^{(j)}}\right)_{i, j=1}^{3}=l_{1} U,\left(\frac{\partial^{2} u(0,0)}{\partial p^{(i)} \partial q^{(j)}}\right)_{i, j=1}^{3}=l U,\left(\frac{\partial^{2} u(0,0)}{\partial q^{(i)} \partial q^{(j)}}\right)_{i, j=1}^{3}=l_{2} U
$$

Remark 2.2. The function $u$ is even and has a unique non-degenerate minimum on $\mathbb{T}^{3}$ and hence without loss of generality we have assumed that the function $u$ has a unique minimum at the point $(0,0) \in\left(\mathbb{T}^{3}\right)^{2}$.

Hypothesis 2.3. The function $\varphi_{\alpha} \in C^{(2)}\left(\mathbb{T}^{3}\right), \alpha=1,2$, is either even or odd on $\mathbb{T}^{3}$.

Set

$$
u_{p}^{(1)}(q)=u(q, p), \quad u_{p}^{(2)}(q)=u(p, q)
$$

To study spectral properties of the operator $H$ we introduce the following two families of bounded self-adjoint operators (the Friedrichs model) $h_{\mu_{\alpha}}(p), p \in \mathbb{T}^{3}$, acting in $L_{2}\left(\mathbb{T}^{3}\right)$ by

$$
h_{\mu_{\alpha}}(p)=h_{\alpha}^{0}(p)-\mu_{\alpha} v_{\alpha}
$$

where

$$
\left(h_{\alpha}^{0}(p) f\right)(q)=u_{p}^{(\alpha)}(q) f(q), \quad f \in L_{2}\left(\mathbb{T}^{3}\right),
$$

and $v_{\alpha}, \alpha=1,2$, are non-local interaction operators

$$
\left(v_{\alpha} f\right)(q)=\varphi_{\alpha}(q) \int_{\mathbb{T}^{3}} \varphi_{\alpha}(t) f(t) d t, \quad f \in L_{2}\left(\mathbb{T}^{3}\right) .
$$

Remark 2.4. The spectrum and resonances of the Friedrichs model are studied in [5, 11 12 26]. 
Let $\mathbb{C}$ be the field of complex numbers. Set

$$
\begin{aligned}
& m_{\alpha}(p)=\min _{q \in \mathbb{T}^{3}} u_{p}^{(\alpha)}(q), \quad M_{\alpha}(p)=\max _{q \in \mathbb{T}^{3}} u_{p}^{(\alpha)}(q), \\
& m=\min _{p, q \in \mathbb{T}^{3}} u(p, q), \quad M=\max _{p, q} u(p, q) .
\end{aligned}
$$

and

$$
\Lambda_{\alpha}(p, z)=\int_{\mathbb{T}^{3}} \frac{\varphi_{\alpha}^{2}(t) d t}{u_{p}^{(\alpha)}(t)-z}, \quad p \in \mathbb{T}^{3}, z \in \mathbb{C} \backslash\left[m_{\alpha}(p), M_{\alpha}(p)\right] .
$$

Remark 2.5. Note that by part (i) of Hypothesis 2.1 all third order partial derivatives of the function $\Lambda_{\alpha}(\cdot, z), z<m$, belong to $\mathcal{B}\left(\theta, \mathbb{T}^{3}\right)$ and if $z=m$, then $\Lambda_{\alpha}(\cdot, m)$ is continuous in $\mathbb{T}^{3}$.

In order to prove the finiteness and infiniteness of eigenvalues below the bottom of the essential spectrum of $H$ we assume the following

Hypothesis 2.6. Assume that the function $\Lambda_{\alpha}(\cdot, m)$ has a unique maximum at the origin such that for some $c>0$ the following inequality holds

$$
\Lambda_{\alpha}(0, m)-\Lambda_{\alpha}(p, m)>c|p|^{2}, \quad 0 \neq p \in U_{\delta}(0) .
$$

Recall (see, e.g., [21, 2]) that a complex-valued bounded function $\varepsilon: \mathbb{T}^{3} \rightarrow \mathbb{C}$ is called conditionally negative definite if $\varepsilon(p)=\overline{\varepsilon(-p)}$ and

$$
\sum_{i, j=1}^{n} \varepsilon\left(p_{i}-p_{j}\right) z_{i} \bar{z}_{j} \leq 0
$$

for any $n \in \mathbb{N}$, for all $p_{1}, p_{2}, \ldots, p_{n} \in \mathbb{T}^{d}$ and all $\mathbf{z}=\left(z_{1}, z_{2}, \ldots, z_{n}\right) \in \mathbb{C}^{n}$ satisfying $\sum_{i=1}^{n} z_{i}=0$.

Remark 2.7. Assume that $\varepsilon(\cdot)$ is a real-valued conditionally negative definite function on $\mathbb{T}^{3}$ having a unique non-degenerate minimum at the origin and such that all third order partial derivatives of $\varepsilon(\cdot)$ are continuous and belong to $\mathcal{B}\left(\theta, \mathbb{T}^{3}\right)$. Let the function $u(\cdot, \cdot)$ be of the form

$$
u(p, q)=\varepsilon(p)+\varepsilon(p-q)+\varepsilon(q) .
$$

Then Hypotheses 2.1] and 2.6 are fulfilled (see Lemma 5.3 in [5]).

Definition 2.8. Let part (i) of Hypothesis 2.1] fulfilled and let $\varphi_{\alpha} \in \mathcal{B}\left(\theta, \mathbb{T}^{3}\right), \frac{1}{2}<\theta \leq$ 1. The operator $h_{\mu_{\alpha}}(0)$ is said to have a threshold energy resonance if the number 1 is an eigenvalue of the operator

$$
\left(\mathrm{G}_{\alpha} \psi\right)(q)=\mu_{\alpha} \varphi_{\alpha}(q) \int_{\mathbb{T}^{3}} \frac{\varphi_{\alpha}(t) \psi(t) d t}{u_{0}^{(\alpha)}(t)-m}, \quad \psi \in C\left(\mathbb{T}^{3}\right)
$$

and the associated eigenfunction $\psi$ (up to constant factor) satisfies the condition $\psi(0) \neq 0$.

Remark 2.9. Let part (i) of Hypothesis 2.1 be fulfilled and let $\varphi_{\alpha} \in \mathcal{B}\left(\theta, \mathbb{T}^{3}\right), \quad \frac{1}{2}<\theta \leq 1$. (i) If $\varphi_{\alpha}(0) \neq 0$ and $\mu_{\alpha}=\mu_{\alpha}^{0}$, then the operator $h_{\mu_{\alpha}^{0}}(0)$ has a threshold energy resonance and the function

$$
f(q)=\frac{\varphi_{\alpha}(q)}{u_{0}^{(\alpha)}(q)-m}
$$

obeys the equation $h_{\mu_{\alpha}^{0}}(0) f=m f$ and $f \in L_{1}\left(\mathbb{T}^{3}\right) \backslash L_{2}\left(\mathbb{T}^{3}\right)$ (see Lemma 3.1).

(ii) If $\varphi_{\alpha}(0)=0$ and $\mu_{\alpha}=\mu_{\alpha}^{0}$, then the operator $h_{\mu_{\alpha}^{0}}(0)$ has a threshold eigenvalue and 
the function $f$, defined by (2.3), obeys the equation $h_{\mu_{\alpha}^{0}}(0) f=m f$ and $f \in L_{2}\left(\mathbb{T}^{3}\right)$ (see Lemma 3.1].

Let $\tau_{\text {ess }}(H)$ be the bottom of the essential spectrum and $N(z)$ be the number of eigenvalues of $H$ lying below $z \leq \tau_{\text {ess }}(H)$.

Set

$$
\mu_{\alpha}^{0}=\Lambda_{\alpha}^{-1}(0, m)
$$

The main results of the present paper are as follows:

Theorem 2.10. Let Hypotheses 2.1] and 2.3 be fulfilled and $\mu_{\alpha}=\mu_{\alpha}^{0}, \alpha=1,2$.

(i) Assume that Hypothesis 2.6 is fulfilled and $\varphi_{1}(0) \varphi_{2}(0)=0$. Then the operator $H$ has a finite number of eigenvalues outside of the essential spectrum.

(ii) Assume $\varphi_{\alpha}(0) \neq 0$ for any $\alpha=1,2$ and that Hypothesis 2.6 is fulfilled. Then the discrete spectrum of $H$ is infinite and the function $N(\cdot)$ obeys the relation

$$
\lim _{z \rightarrow m-0} \frac{N(z)}{|\log | m-z||}=\mathfrak{U}_{0} \quad\left(0<\mathfrak{U}_{0}<\infty\right) .
$$

Remark 2.11. In fact in [5] a result analogue, to part (i) of Theorem 2.10 has been proven for the three-particle Schrödinger operators on the lattice $\mathbb{Z}^{3}$ in the case, where the function $u(\cdot, \cdot)$ is of the form

$$
u(p, q)=\varepsilon(p)+\varepsilon(p-q)+\varepsilon(q),
$$

and

$$
\varepsilon(q)=3-\cos _{1}-\cos _{2}-\cos _{3}, \quad q=\left(q_{1}, q_{2}, q_{3}\right) \in \mathbb{T}^{3},
$$

$\varphi_{\alpha}(\cdot) \equiv$ const.

Remark 2.12. The constant $\mathcal{U}_{0}$ does not depend on the functions $\varphi_{\alpha}$ and is given as a positive function depending only on the ratios $\frac{l_{\alpha}}{l}, \alpha=1,2$.

Remark 2.13. Clearly, the infinite cardinality of the discrete spectrum of $H$ lying on the l.h.s. of $m$ follows automatically from the positivity of $\mathcal{U}_{0}$.

Remark 2.14. Notice that under assumptions of Theorem 2.10 for all nonzero $p \in \mathbb{T}^{3}$ the operator $h_{\mu_{\alpha}^{0}}(p)-m I$ is strictly positive and so that the operator $h_{\mu_{\alpha}^{0}}(0)$ corresponding to zero value of $p$ is a unique operator whose spectrum attains the bottom of the essential spectrum of $H$. Moreover the essential spectrum $\sigma_{e s s}(H)$ of $H$ consists only of the threeparticle continuиm $[m, M]$.

\section{THRESHOLD ANALYSIS OF THE FAMILY OF FRIEDRICHS MODELS $h_{\mu_{\alpha}}(p)$}

In this section for the reader's convenience we recall some results concerning the families of Friedrichs models $h_{\mu_{\alpha}}(p), p \in \mathbb{T}^{3}$, defined by [2.2), from the paper [5].

In accordance to Weyl's theorem the essential spectrum of the operator $h_{\mu_{\alpha}}(p)$ fills the following interval on the real axis:

$$
\sigma_{e s s}\left(h_{\mu_{\alpha}}(p)\right)=\left[m_{\alpha}(p), M_{\alpha}(p)\right] .
$$

For any $p \in \mathbb{T}^{3}$ we define an analytic function $\Delta_{\mu_{\alpha}}(p, \cdot)$ (the Fredholm determinant associated to the operator $\left.h_{\mu_{\alpha}}(p)\right)$ on $\mathbb{C} \backslash\left[m_{\alpha}(p), M_{\alpha}(p)\right]$ by

$$
\Delta_{\mu_{\alpha}}(p, z)=1-\mu_{\alpha} \Lambda_{\alpha}(p, z) .
$$

The following lemma describes whether the bottom of the essential spectrum of $h_{\mu_{\alpha}^{0}}(0)$ is a threshold energy resonance or a threshold eigenvalue. 
Lemma 3.1. Assume part (i) of Hypothesis 2.1] and $\varphi_{\alpha} \in \mathcal{B}\left(\theta, \mathbb{T}^{3}\right)$.

(i) For any $\mu_{\alpha}>0$ and $p \in \mathbb{T}^{3}$ the operator $h_{\mu_{\alpha}}(p)$ has an eigenvalue $z \in \mathbb{C} \backslash$ $\left[m_{\alpha}(p), M_{\alpha}(p)\right]$ if and only if $\Delta_{\mu_{\alpha}}(p, z)=0$.

(ii) the operator $h_{\mu_{\alpha}}(0)$ has a threshold energy resonance (resp. threshold eigenvalue) if and only if $\mu_{\alpha}=\mu_{\alpha}{ }^{0}$ and $\varphi_{\alpha}(0) \neq 0\left(\right.$ resp. $\left.\varphi_{\alpha}(0)=0\right)$.

The following Lemma 3.2 plays a crucial role in the proof of the infiniteness (resp. finiteness) of the number of eigenvalues lying below the bottom of the essential spectrum for a model operator $H$ associated to a system of three-particles on the lattice $\mathbb{Z}^{3}$ interacting via pair non-local potential.

Lemma 3.2. Assume that Hypotheses 2.1] and 2.3 are fulfilled.

(i) Let the operator $h_{\mu_{\alpha}^{0}}(0)$ have a threshold energy resonance. Then:

$\left(i_{1}\right)$ for all $p \in U_{\delta}(0)$ and $z \leq m$ the following expansion holds

$$
\Delta_{\mu_{\alpha}^{0}}(p, z)=\frac{4 \sqrt{2} \pi^{2} \mu_{\alpha}^{0} \varphi_{\alpha}^{2}(0)}{l_{\beta}^{\frac{3}{2}} \operatorname{det}(U)^{\frac{1}{2}}} \sqrt{m_{\alpha}(p)-z}+\Delta_{\mu_{\alpha}^{0}}^{r e s}(z)+\Delta_{\mu_{\alpha}^{0}}^{r e s}(p, z),
$$

where $\Delta_{\mu}^{r e s}\left(m_{\alpha}(p)-z\right)=O\left(\left(m_{\alpha}(p)-z\right)^{\frac{1+\theta}{2}}\right)$ as $m_{\alpha}(p)-z \rightarrow 0, z<m_{\alpha}(p)$, and $\Delta_{\mu_{\alpha}}^{\text {res }}(p, z)=O\left(p^{2}\right)$ as $p \rightarrow 0$ uniformly in $z \leq m_{\alpha}(p)$;

$\left(i_{2}\right)$ for some $c_{1}, c_{2}>0$ the inequalities

$$
\begin{gathered}
c_{1}|p| \leq \Delta_{\mu_{\alpha}^{0}}(p, m) \leq c_{2}|p|, \quad p \in U_{\delta}(0), \\
\Delta_{\mu_{\alpha}^{0}}(p, m) \geq c, \quad p \in \mathbb{T}^{3} \backslash U_{\delta}(0)
\end{gathered}
$$

hold.

(ii) Let $z=m$ be an eigenvalue of $h_{\mu_{\alpha}^{0}}(0)$. Then:

(ii $\left.i_{1}\right)$ for any $p \in U_{\delta}(0)$ and $z \leq m$ the following expansion holds

$$
\Delta_{\mu_{\alpha}^{0}}(p, z)=\Delta_{\mu_{\alpha}^{0}}^{r e s}(z)+\Delta_{\mu_{\alpha}^{0}}^{r e s}(p, z)
$$

$\left(i i_{2}\right)$ the inequality

$$
\Delta_{\mu_{\alpha}^{0}}(p, m) \geq c p^{2}, \quad p \in U_{\delta}(0)
$$

holds, for some $c>0$.

Remark 3.3. Lemma 3.2 gives threshold energy expansions for the Fredholm determinant, leading to different behaviors for a threshold energy resonance resp. eigenvalue.

\section{THE ESSENTIAL SPECTRUM OF THE OPERATOR $H$}

In this section we exhibit the location of the essential spectrum of the model operator $H$.

Set

$$
\Sigma=\cup_{\alpha=1}^{2} \cup_{p \in \mathbb{T}^{3}} \sigma_{d}\left(h_{\mu_{\alpha}}(p)\right) \cup[m, M],
$$

where $\sigma_{d}\left(h_{\mu_{\alpha}}(p)\right)$ is the discrete spectrum of the operator $h_{\mu_{\alpha}}(p), p \in \mathbb{T}^{3}$.

Let $\Phi_{\alpha}: L_{2}\left(\left(\mathbb{T}^{3}\right)^{2}\right) \rightarrow L_{2}\left(\mathbb{T}^{3}\right), \alpha=1,2$, be the operator defined by

$$
\left(\Phi_{1} f\right)(q)=\int_{\mathbb{T}^{3}} \varphi_{1}(t) f(t, q) d t, \quad\left(\Phi_{2} f\right)(p)=\int_{\mathbb{T}^{3}} \varphi_{2}(t) f(p, t) d t, f \in L_{2}\left(\left(\mathbb{T}^{3}\right)^{2}\right)
$$

and denote by $\Phi_{\alpha}^{*}$ its adjoint. Let $D_{\alpha}(z)$ be the multiplication operator by the function $\Delta_{\mu_{\alpha}}(q, z)$ on $L_{2}\left(\mathbb{T}^{3}\right)$. 
It is easy to prove the equality

$$
I-\mu_{\alpha} \Phi_{\alpha} R_{0}(z) \Phi_{\alpha}^{*}=D_{\alpha}(z), \quad z \in \mathbb{C} \backslash[m, M],
$$

where $R_{0}(z)=\left(H_{0}-z \mathbf{I}\right)^{-1}$ is the resolvent of $H_{0}$ and $I$ resp. $\mathbf{I}$ is the identity operator on $L_{2}\left(\mathbb{T}^{3}\right)$ resp. $L_{2}\left(\left(\mathbb{T}^{3}\right)^{2}\right)$.

By Lemma3.1 for any $z \in \mathbb{C} \backslash \Sigma$ the inequality $\Delta_{\mu_{\alpha}}(p, z) \neq 0$ holds. Then the operator $D_{\alpha}(z), z \in \mathbb{C} \backslash \Sigma$, is invertible. Let $D_{\alpha}^{-1}(z)$ be its inverse.

Let $\mathrm{T}(z), z \in \mathbb{C} \backslash \Sigma$, act in $L_{2}^{(2)}\left(\mathbb{T}^{3}\right)$ with the entries

$$
\mathrm{T}_{\alpha \alpha}(z)=0, \quad \mathrm{~T}_{\alpha \beta}(z)=\sqrt{\mu_{\alpha} \mu_{\beta}} D_{\alpha}^{-1}(z) \Phi_{\alpha} R_{0}(z) \Phi_{\beta}^{*} .
$$

Lemma 4.1. For any $z \in \mathbb{C} \backslash \Sigma$ the operator $\mathrm{T}(z)$ is an Hilbert-Schmidt operator.

Proof. According to the fact that $\Phi_{\alpha} \Phi_{\beta}^{*}, \alpha \neq \beta$, is a compact integral operator one checks that for any $z \in \mathbb{C} \backslash \Sigma$ the operator $\Phi_{\alpha} R_{0}(z) \Phi_{\beta}^{*}$ belongs to the Hilbert-Schmidt class $\Sigma_{2}$. Since the operator $D_{\alpha}^{-1}(z), z \in \mathbb{C} \backslash \Sigma$, is bounded, the operator $\mathrm{T}_{\alpha \beta}(z)$ also belongs to $\Sigma_{2}$.

The following theorem describes the essential spectrum of the operator $H$ by the spectrum of the family $h_{\mu_{\alpha}}(p), p \in \mathbb{T}^{3}, \alpha=1,2$.

Theorem 4.2. For the essential spectrum $\sigma_{\text {ess }}(H)$ of the operator $H$ the following equality holds

$$
\sigma_{e s s}(H)=\cup_{\alpha=1}^{2} \cup_{p \in \mathbb{T}^{3}} \sigma_{d}\left(h_{\mu_{\alpha}}(p)\right) \cup[m, M] .
$$

Proof. The proof of theorem consists of two steps. The inclusion $\Sigma \subset \sigma_{\text {ess }}(H)$ is proven using Weyl's criterion, as given in [4] (we omit the details). Let us prove the inclusion $\sigma_{\text {ess }}(H) \subset \Sigma$.

Denote by $R(z)=(H-z \mathbf{I})^{-1}$ the resolvent of the operator $H$. The well known resolvent equation has the form

$$
R(z)=R_{0}(z)+R_{0}(z)\left(\mu_{1} V_{1}+\mu_{2} V_{2}\right) R(z) .
$$

We observe that $V_{\alpha}=\Phi_{\alpha}^{*} \Phi_{\alpha}$. Multiplying (4.2) from the left side by $\sqrt{\mu_{\alpha}} \Phi_{\alpha}$ and setting $\mathcal{R}_{\alpha}(z) \equiv \sqrt{\mu_{\alpha}} \Phi_{\alpha} R(z)$ we get the system of equations

$$
\mathcal{R}_{\alpha}(z)=\sqrt{\mu_{\alpha}} \Phi_{\alpha} R_{0}(z)+\sqrt{\mu_{\alpha}} \Phi_{\alpha} R_{0}(z)\left(\sqrt{\mu_{1}} \Phi_{1}^{*} \mathcal{R}_{1}(z)+\sqrt{\mu_{2}} \Phi_{2}^{*} \mathcal{R}_{2}(z)\right), \alpha=1,2,
$$

or the following system of three equations

$$
\left(I-\mu_{\alpha} \Phi_{\alpha} R_{0}(z) \Phi_{\alpha}^{*}\right) \mathcal{R}_{\alpha}(z)=\sqrt{\mu_{\alpha}} \Phi_{\alpha} R_{0}(z)+\sqrt{\mu_{\alpha} \mu_{\beta}} \Phi_{\alpha} R_{0}(z) \Phi_{\beta}^{*} \mathcal{R}_{\beta}(z), \alpha=1,2 .
$$

As we mentioned above (4.1) the operator $\left(I-\mu_{\alpha} \Phi_{\alpha} R_{0}(z) \Phi_{\alpha}^{*}\right) \equiv D_{\alpha}(z), z \in \mathbb{C} \backslash \Sigma$, is a multiplication operator on $L_{2}\left(\mathbb{T}^{3}\right)$ and is invertible.

Multiplying the equality (4.3) from the left by $D_{\alpha}^{-1}(z)$ we get the Faddeev type equation

$$
\mathcal{R}(z)=\mathcal{R}_{0}(z)+\mathrm{T}(z) \mathcal{R}(z),
$$

where $\mathcal{R}(z)=\left(\mathcal{R}_{1}(z), \mathcal{R}_{2}(z)\right)$ and $\mathcal{R}_{0}(z)=\left(\sqrt{\mu_{1}} D_{1}^{-1}(z) \Phi_{1} R_{0}(z), \sqrt{\mu_{2}} D_{2}^{-1}(z) \Phi_{2} R_{0}(z)\right)$ are vector operators.

From (4.2) we have the following representation for the resolvent

$$
R(z)=R_{0}(z)+R_{0}(z)\left(\sqrt{\mu_{1}} \Phi_{1}^{*} \mathcal{R}_{1}(z)+\sqrt{\mu_{2}} \Phi_{2}^{*} \mathcal{R}_{2}(z)\right) .
$$

Let $\mathcal{J}$ be the identity operator in $L_{2}^{(2)}\left(\mathbb{T}^{3}\right)$. Since $\|\mathrm{T}(z)\| \rightarrow 0$ as $z \rightarrow \infty$ the operator $\mathrm{T}(z)$ is a compact operator-valued function on $\mathbb{C} \backslash \Sigma$ and $\mathcal{J}-\mathrm{T}(z)$ is invertible if $z$ is 
real and either very negative or very positive. The analytic Fredholm theorem (see, e.g., Theorem VI.14 in [21]) implies that there is a discrete set $S \subset \mathbb{C} \backslash \Sigma$ so that $(\mathcal{J}-\mathrm{T}(z))^{-1}$ exists and is analytic in $\mathbb{C} \backslash(\Sigma \cup S)$ and meromorphic in $\mathbb{C} \backslash \Sigma$ with finite rank residues. Thus the function $(\mathcal{J}-\mathrm{T}(z))^{-1} \mathcal{R}_{0}(z) \equiv F(z)$ is analytic in $\mathbb{C} \backslash(\Sigma \cup S)$ with finite rank residues at the points of $S$.

Let $z \notin S, \operatorname{Im} z \neq 0$, then by 4.4, 4.5 we have $F(z)=\mathcal{R}(z)$. In particular,

$$
R(z)(H-z \mathbf{I})=\left(R_{0}(z)+R_{0}(z) \sum_{\alpha=1}^{2} \sqrt{\mu_{\alpha}} \Phi_{\alpha}^{*} \mathcal{R}_{\alpha}(z)\right)(H-z \mathbf{I})=\mathbf{I} .
$$

By analytic continuation, this holds for any $z \notin \Sigma \cup S$. We conclude that, for any such $z$, the operator $H-z \mathbf{I}$ has a bounded inverse. Therefore $\sigma(H) \backslash \Sigma$ consists of isolated points and only the frontier points of $\Sigma$ can possibly be limit points. Finally, since $R(z)$ has finite rank residues at $z \in S$, we conclude that $\sigma(H) \backslash \Sigma$ belongs to the discrete spectrum $\sigma_{d}(H)$ of $H$, which completes the proof.

Corollary 4.3. Let the assumptions of Theorem 2.10 be fulfilled. Then the essential spectrum $\sigma_{\text {ess }}(H)$ of $H$ consists of the interval $[m, M]$.

Proof. Hypothesis 2.6 and the equality $\mu_{\alpha}^{0}=\Lambda_{\alpha}^{-1}(0, m)$ yield $\Delta_{\mu_{\alpha}^{0}}(0, m)=0$ and hence the inequality

$$
\Delta_{\mu_{\alpha}^{0}}(p, m)=\mu_{\alpha}^{0}\left(\Lambda_{\alpha}(0, m)-\Lambda_{\alpha}(p, m)\right)>0, \quad 0 \neq p \in \mathbb{T}^{3} .
$$

Since the function $\Delta_{\mu_{\alpha}^{0}}(p, \cdot)$ is decreasing in $\left(-\infty, m_{\alpha}(p)\right)$ by Lemma 3.1 we obtain $h_{\mu_{\alpha}^{0}}(p)>m, 0 \neq p \in \mathbb{T}^{3}$. This argument, together with Theorem 4.2 completes the proof of Corollary 4.3

4.1. Birman-Schwinger principle. We recall that $\tau_{\text {ess }}(H)$ denotes the bottom of the essential spectrum and $N(z)$ the number of eigenvalues of $H$ lying below $z \leq \tau_{\text {ess }}(H)$.

For a bounded self-adjoint operator $B$, we define $n(\lambda, B)$ by

$$
n(\lambda, B)=\sup \{\operatorname{dim} F:(B u, u)>\lambda, u \in F,\|u\|=1\} .
$$

$n(\lambda, B)$ is equal to infinity if $\lambda$ is in the essential spectrum of $B$ and if $n(\lambda, B)$ is finite, it is equal to the number of the eigenvalues of $B$ larger than $\lambda$. By the definition of $N(z)$ we have

$$
N(z)=n(-z,-H),-z>-\tau_{e s s}(H) .
$$

In our analysis of the spectrum of $H$ the crucial role is played by the self-adjoint compact operator $T(z), z<\tau_{\text {ess }}(H)$ in $L_{2}^{(2)}\left(\mathbb{T}^{3}\right)$ with the entries

$$
T_{\alpha \alpha}(z)=0, \quad T_{\alpha \beta}(z)=\sqrt{\mu_{1} \mu_{2}} D_{\alpha}^{-\frac{1}{2}}(z) \Phi_{\alpha} R_{0}(z) \Phi_{\beta}^{*} D_{\beta}^{-\frac{1}{2}}(z) .
$$

The following lemma is a realization of the well known Birman-Schwinger principle for the operator $H$ (see [3, 22, 24] ).

Lemma 4.4. The operator $T(z)$ is compact and continuous in $z<\tau_{\text {ess }}(H)$ and

$$
N(z)=n(1, T(z)) \text {. }
$$

Proof. This lemma is deduced by the same arguments as well as in [3] 22]. Set $V=$ $\mu_{1} V_{1}+\mu_{2} V_{2}$. Since for any $z<\tau_{\text {ess }}(H)$ the following relation

$$
\begin{aligned}
& f \in L_{2}\left(\left(\mathbb{T}^{3}\right)^{2}\right),(H f, f)<z(f, f) \Leftrightarrow\left(R_{0}^{\frac{1}{2}}(z) V R_{0}^{\frac{1}{2}}(z) g, g\right)>(g, g), \\
& g=\left(R_{0}(z)\right)^{-\frac{1}{2}} f, g \in L_{2}\left(\left(\mathbb{T}^{3}\right)^{2}\right),
\end{aligned}
$$


holds, the quantity $N(z)$ in question coincides with $n\left(1, R_{0}^{\frac{1}{2}}(z) V R_{0}^{\frac{1}{2}}(z)\right)$, that is,

$$
N(z)=n\left(1, R_{0}^{\frac{1}{2}}(z) V R_{0}^{\frac{1}{2}}(z)\right) .
$$

Decompose $\left.R_{0}^{\frac{1}{2}}(z) V R_{0}^{\frac{1}{2}}(z)\right)=B^{*} B$, with the vector operator $B: L_{2}\left(\left(\mathbb{T}^{3}\right)^{2}\right) \rightarrow$ $L_{2}^{(2)}\left(\mathbb{T}^{3}\right)$ defined by

$$
B=\left(\sqrt{\mu_{1}} \Phi_{1} R_{0}^{\frac{1}{2}}(z), \sqrt{\mu_{2}} \Phi_{2} R_{0}^{\frac{1}{2}}(z)\right) .
$$

One can see that the operator $M(z)=B B^{*}$ acts in $L_{2}^{(2)}\left(\mathbb{T}^{3}\right)$ with the entries

$$
M_{\alpha \beta}(z)=\sqrt{\mu_{\alpha} \mu_{\beta}} \Phi_{\alpha} R_{0}(z) \Phi_{\beta}^{*}, \quad \alpha, \beta=1,2 .
$$

Both operators $B^{*} B$ and $M(z)=B B^{*}$ have the same nonzero eigenvalues with the same multiplicities. We use this argument to obtain the equality

$$
N(z)=n(1, M(z)) \text {. }
$$

We decompose $M(z)$ into the sum $M(z)=M_{0}(z)+K(z)$, where

$$
M_{0}(z)=\operatorname{diag}\left\{M_{11}(z), M_{22}(z)\right\}, \quad K(z)=\left(\begin{array}{cc}
0 & M_{12}(z) \\
M_{21}(z) & 0
\end{array}\right) .
$$

By (4.1) the operator $I-M_{\alpha \alpha}(z)=D_{\alpha}(z), z<\tau_{\text {ess }}(H)$, is invertible and by $\Delta_{\mu_{\alpha}^{0}}(p, z)>0, p \in \mathbb{T}^{3}, z<\tau_{\text {ess }}(H)$, it is positive and a direct calculation shows that $n(1, M(z))=n\left(1,\left(\mathcal{J}-M_{0}(z)\right)^{-\frac{1}{2}} K(z)\left(\mathcal{J}-M_{0}(z)\right)^{-\frac{1}{2}}\right)$. Then, to finish the proof, it suffices to coincide the equality $T(z)=\left(\mathcal{J}-M_{0}(z)\right)^{-\frac{1}{2}} K(z)\left(\mathcal{J}-M_{0}(z)\right)^{-\frac{1}{2}}$ and (4.6).

\section{THE FINITENESS OF THE NUMBER OF EIGENVALUES OF THE OPERATOR $H$.}

In this section we will prove part (i) of Theorem 2.10 (the finiteness of the number of eigenvalues ). We starts the proof with the following assertion

Theorem 5.1. The operator $H$ has no eigenvalues lying on the right hand side of the essential spectrum $\sigma_{\text {ess }}(H)$.

Proof. Since $V=\mu_{1} V_{1}+\mu_{2} V_{2}$ is a positive operator and $\sup \left(\sigma_{\text {ess }}(H)\right)=\sup \left(\sigma\left(H_{0}\right)\right)=$ $M$ we have that the operator $H=H_{0}-V$ has no eigenvalues larger than $M$.

Now we prove that $H$ has a finite number of eigenvalues on the left hand side of its essential spectrum.

To do this, we use the following two lemmas.

Lemma 5.2. Let Hypothesis 2.1 be fulfilled. Then there exist numbers $C_{1}, C_{2}, C_{3}>0$ and $\delta>0$ such that the following inequalities hold

(i) $\quad C_{1}\left(|p|^{2}+|q|^{2}\right) \leq u(p, q)-m \leq C_{2}\left(|p|^{2}+|q|^{2}\right) \quad$ for all $\quad p, q \in U_{\delta}(0)$,

(ii) $u(p, q)-m \geq C_{3}$ for all $(p, q) \notin U_{\delta}(0) \times U_{\delta}(0)$.

Proof. By Hypothesis 2.1 the point $(0,0) \in\left(\mathbb{T}^{3}\right)^{2}$ is a unique non-degenerated minimum point of $u$. Then there exist positive numbers $C_{1}, C_{2}, C_{3}$ and a $\delta$-neighborhood of $(p, q)=$ $(0,0) \in\left(\mathbb{T}^{3}\right)^{2}$ so that $(i)$ and $(i i)$ hold true.

Lemma 5.3. Let the conditions in part $(i)$ of Theorem 2.10 be fulfilled. Then the operator $T(z)$ belongs to the Hilbert-Schmidt class and is continuous from the left up to $z=m$. 
Proof. We prove Lemma5.3 in the case $\mu_{\alpha}=\mu_{\alpha}^{0}$, and $\varphi_{1}(0)=0, \varphi_{2}(0) \neq 0$ (the other cases are handled in a similar way).

Since the function $\varphi_{1} \in C^{(2)}\left(\mathbb{T}^{3}\right)$ is either even or odd and $\varphi_{1}(0)=0$ we have $\left|\varphi_{1}(p)\right| \leq C|p|$ for any $p \in \mathbb{T}^{3}$ and for some $C>0$. By virtue of Lemmas 3.2 and 5.2 the kernel of the operator $T_{12}(z), z \leq m$, is estimated by

$$
C\left(\frac{\chi_{\delta}(p)}{|p|}+1\right)\left(\frac{|q| \chi_{\delta}(p) \chi_{\delta}(q)}{p^{2}+q^{2}}+1\right)\left(\frac{\chi_{\delta}(q)}{|q|+1}\right),
$$

where $\chi_{\delta}(p)$ is the characteristic function of $U_{\delta}(0)$.

Since the function is square-integrable on $\left(\mathbb{T}^{3}\right)^{2}$ we have that $T_{12}(z)$ and $T_{21}(z)=$ $T_{12}^{*}(z)$ are Hilbert-Schmidt operator.

The kernel function of $T_{\alpha \beta}(z)$ is continuous in $p, q \in \mathbb{T}^{3}$ and in $z<m$ and is squareintegrable on $\left(\mathbb{T}^{3}\right)^{2}$ as $z \leq m$. Now the continuity of the operator $T_{\alpha \beta}(z)$ from the left up to $z=m$ follow using Lebesgue's dominated convergence theorem.

We are now ready for the

Proof of $(i)$ of Theorem 2.10 Let the conditions in part $(i)$ of Theorem 2.10 be fulfilled. By Lemma 4.4 we have

$$
N(z)=n(1, T(z)) \text { as } z<m
$$

and by Lemma 5.3 for any $\gamma \in[0,1)$ the number $n(1-\gamma, T(m))$ is finite. Then for all $z<m$ and $\gamma \in(0,1)$ we have

$$
N(z)=n(1, T(z)) \leq n(1-\gamma, T(m))+n(\gamma, T(z)-T(m)) .
$$

This relation can easily be obtained by using Weyl's inequality

$$
n\left(\lambda_{1}+\lambda_{2}, A_{1}+A_{2}\right) \leq n\left(\lambda_{1}, A_{1}\right)+n\left(\lambda_{2}, A_{2}\right)
$$

for the sum of compact operators $A_{1}$ and $A_{2}$ and for positive numbers $\lambda_{1}$ and $\lambda_{2}$.

By Lemma 5.3 the operator $T(z)$ is continuous from the left up to $z=m$ and hence

$$
\lim _{z \rightarrow m-0} N(z)=N(m) \leq n(1-\gamma, T(m)) \text { for all } \gamma \in(0,1) .
$$

Thus

$$
N(m) \leq n(1-\gamma, T(m))<\infty .
$$

The latter inequality and Theorem 5.1 prove the assertion $(i)$ of Theorem 2.10

\section{ASYMPTOTICS FOR THE NUMBER OF EIGENVALUES OF THE OPERATOR $H$.}

In this section we shall derive the asymptotics 2.4) for the number of eigenvalues of $H$. By Hypothesis 2.1 we get

$$
u(p, q)=m+\frac{1}{2}\left(l_{1}(U p, p)+2 l(U p, q)+l_{2}(U q, q)\right)+O\left(|p|^{3+\theta}+|q|^{3+\theta}\right)
$$

as $p, q \rightarrow 0$ and

$$
m_{\alpha}(p)=m+\frac{l_{1} l_{2}-l^{2}}{2 l_{\beta}}(U p, p)+O\left(|p|^{3+\theta}\right) \quad \text { as } \quad p \rightarrow 0 .
$$

Applying the latter asymptotics for $m_{\alpha}(p)$ and using Lemma 3.2 we have

$$
\Delta_{\mu_{\alpha}^{0}}(p, z)=\frac{4 \pi^{2} \mu_{\alpha}^{0} \varphi_{\alpha}^{2}(0)}{l_{\beta}^{3 / 2} \operatorname{det}(U)^{\frac{1}{2}}}\left[n_{\alpha}(U p, p)-2(m-z)\right]^{\frac{1}{2}}+O\left(\left(|p|^{2}+|m-z|\right)^{\frac{1+\theta}{2}}\right)
$$

as $p,|m-z| \rightarrow 0$, where

$$
n_{\alpha}=\left(l_{1} l_{2}-l^{2}\right) / l_{\beta}
$$


Let $T(\delta ;|m-z|)$ be the integral operator in $L_{2}^{(2)}\left(\mathbb{T}^{3}\right)$ with the kernel $T_{\alpha \alpha}(\delta,|m-z| ; p, q)=0$,

$T_{\alpha \beta}(\delta,|m-z| ; p, q)=\mathrm{d}_{0} \frac{\hat{\chi}_{\delta}(p) \hat{\chi}_{\delta}(q)\left(n_{\alpha}(U p, p)+2|m-z|\right)^{-\frac{1}{4}}\left(n_{\beta}(U q, q)+2|m-z|\right)^{-\frac{1}{4}}}{l_{\alpha}(U p, p)+2 l(U p, q)+l_{\beta}(U q, q)+2|m-z|}$, where $\hat{\chi}_{\delta}(\cdot)$ is the characteristic function of the region $\hat{U}_{\delta}(0)=\left\{p \in \mathbb{T}^{3}:\left|U^{\frac{1}{2}} p\right|<\delta\right\}$ and

$$
\mathrm{d}_{0}=\frac{\operatorname{det} U^{\frac{1}{2}}}{2 \pi^{2}} l_{1}^{\frac{3}{4}} l_{2}^{\frac{3}{4}} .
$$

Lemma 6.1. Let the conditions in part (ii) of Theorem 2.10 be fulfilled. The operator $T(z)-T(\delta ;|m-z|)$ belongs to the Hilbert-Schmidt class and is continuous in $z \leq m$.

Proof. Applying asymptotics (6.1), 6.2) and Lemmas 3.2 5.2 one can estimate the kernel of the operator $T_{\alpha \beta}(z)-T_{\alpha \beta}(\delta ;|m-z|), z \leq m$ by the square-integrable function

$$
C\left(\frac{|p|^{1+\theta}+|q|^{1+\theta}}{|p|^{\frac{1}{2}}\left(p^{2}+q^{2}\right)|q|^{\frac{1}{2}}}+\frac{|m-z|^{\frac{\theta}{2}}\left(p^{2}+q^{2}\right)^{-1}}{\left(|p|^{2}+|m-z|\right)^{\frac{1}{4}}\left(|q|^{2}+|m-z|\right)^{\frac{1}{4}}}+1\right) .
$$

Hence the operator $T_{\alpha \beta}(z)-T_{\alpha \beta}(\delta ;|m-z|)$ belongs to the Hilbert-Schmidt class for all $z \leq m$. In combination with the continuity of the kernel of the operator in $z<m$ this gives the continuity of $T(z)-T(\delta ;|m-z|)$ in $z \leq m$.

Let us now recall some results from [22], which are important in our work.

Set $\sigma=L_{2}\left(\mathbb{S}^{2}\right)$, where $\mathbb{S}^{2}$ being unit sphere in $\mathbb{R}^{3}$, and $\sigma^{(2)}=\sigma \oplus \sigma$.

Let $\mathbf{S}_{\mathbf{r}}, \mathbf{r}>0$, be the integral operator in $L_{2}\left((0, \mathbf{r}), \sigma^{(2)}\right)$ with the kernel $S_{\alpha \beta}(y, t), y=$ $x-x^{\prime}, x, x^{\prime} \in(0, \mathbf{r}), t=<\xi, \eta>, \xi, \eta \in \mathbb{S}^{2}$, where

$$
\begin{gathered}
S_{\alpha \alpha}(y, t)=0, \quad S_{\alpha \beta}(y, t)=(2 \pi)^{-2} \frac{u_{\alpha \beta}}{\cosh \left(y+r_{\alpha \beta}\right)+s_{\alpha \beta} t}, \\
u_{\alpha \beta}=u_{\beta \alpha}=\left(\frac{l_{1} l_{2}}{l_{1} l_{2}-l^{2}}\right)^{\frac{1}{2}}, r_{\alpha \beta}=\frac{1}{2} \log \frac{l_{\alpha}}{l_{\beta}}, s_{\alpha \beta}=s_{\beta \alpha}=\frac{l}{\sqrt{l_{1} l_{2}}}, \\
\alpha, \beta=1,2 .
\end{gathered}
$$

Let $\hat{\mathbf{S}}(\lambda), \lambda \in \mathbb{R}$, be the integral operator on $\sigma^{(2)}$ whose kernel depends on the scalar product $t=<\xi, \eta>$ of the arguments $\xi, \eta \in \mathbb{S}^{2}$ and has the form

$$
\hat{S}_{\alpha \alpha}(\lambda ; t)=0, \quad \hat{S}_{\alpha \beta}(\lambda ; t)=(2 \pi)^{-2} \frac{u_{\alpha \beta} e^{i r_{\alpha \beta} \lambda} \sinh \left[\lambda\left(\operatorname{arccoss}_{\alpha \beta} t\right)\right]}{\sqrt{1-s_{\alpha \beta}^{2} t} \sinh (\pi x)} .
$$

For $\mu>0$, define

$$
U(\mu)=(4 \pi)^{-1} \int_{-\infty}^{+\infty} n(\mu, \hat{\mathbf{S}}(\lambda)) d \lambda
$$

This function was studied in detail [22] and is very important for the proof of the existence of the Efimov effect. In particular, it was proved that $U(\cdot)$ is continuous, $U(1)>0$ if $u_{12}>1$ and

$$
\lim _{\mathbf{r} \rightarrow \infty} \frac{1}{2} \mathbf{r}^{-1} n\left(\mu, \mathbf{S}_{\mathbf{r}}\right)=U(\mu) .
$$

Part (ii) of Theorem 2.10 will be deduced by a perturbation argument based on the following lemma (see Lemma 4.7 in [22]). For completeness, we reproduce the proof given there. 
Lemma 6.2. Let $A(z)=A_{0}(z)+A_{1}(z)$, where $A_{0}(z)$ resp. $A_{1}(z)$ is compact and continuous in $z<m$ resp. $z \leq m$. Assume that for some function $f(\cdot), f(z) \rightarrow 0, z \rightarrow$ $m-0$ the limit

$$
\lim _{z \rightarrow m-0} f(z) n\left(\lambda, A_{0}(z)\right)=l(\lambda)
$$

exists and $l(\lambda)$ is continuous in $\lambda>0$. Then the same limit exists for $A(z)$ and

$$
\lim _{z \rightarrow m-0} f(z) n(\lambda, A(z))=l(\lambda) .
$$

Proof. According to Weyl's inequality (5.1) for any $\varepsilon \in(0,1)$ we have

$$
\begin{gathered}
n(\lambda, A(z)) \leq n\left((1-\varepsilon) \lambda, A_{0}(z)\right)+n\left(\varepsilon \lambda, A_{1}(z)\right), \\
n(\lambda, A(z)) \geq n\left((1+\varepsilon) \lambda, A_{0}(z)\right)-n\left(\varepsilon \lambda, A_{1}(z)\right)
\end{gathered}
$$

Since the operator $A_{1}(z)$ is compact and continuous in $z \leq m$, we obtain

$$
l((1+\varepsilon) \lambda) \leq \lim _{z \rightarrow m-0} \inf f(z) n(\lambda, A(z)) \leq \lim _{z \rightarrow m-0} \sup f(z) n(\lambda, A(z)) \leq l((1-\varepsilon)) .
$$

Then the continuity of the function $l(\lambda)$ in $\lambda>0$ completes the proof of Lemma 6.2

Remark 6.3. Since $U(\cdot)$ is continuous in $\mu$, according to Lemma 6.2 any perturbations of the operator $A_{0}(z)$ defined in Lemma 6.2 which is compact and continuous up to $z=m$ do not contribute to asymptotics 2.4. During the proof of Theorem 6.4 we use this fact without further comments.

The following theorem is basic for the proof of the asymptotics 2.4.

Theorem 6.4. Let the conditions in part (i) of Theorem 2.10 be fulfilled. Then the equality

$$
\lim _{|m-z| \rightarrow 0}|\log | z-m||^{-1} n(\mu, T(\delta ;|m-z|))=U(\mu), \quad \mu>0,
$$

holds.

Proof. The space of vector-functions $w=\left(w_{1}, w_{2}\right)$ with coordinates having support in $\hat{U}_{\delta}(0)$ is an invariant subspace for the operator $T(\delta|m-z|)$.

Let $\hat{T}_{0}(\delta ;|m-z|)$ be the restriction of the integral operator $T(\delta|m-z|)$ to the subspace $L_{2}^{(2)}\left(\hat{U}_{\delta}(0)\right)$. One verifies that the operator $\hat{T}_{0}(\delta ;|m-z|)$ is unitarily equivalent to the integral operator $T_{0}(\delta ;|m-z|)$ in $L_{2}^{(2)}\left(\hat{U}_{\delta}(0)\right)$ with the kernel

$$
\begin{aligned}
& T_{\alpha \alpha}^{(0)}(\delta,|m-z| ; p, q)=0, \\
& T_{\alpha \beta}^{(0)}(\delta,|m-z| ; p, q)=\mathrm{d}_{1} \frac{\left(n_{\alpha} p^{2}+2|m-z|\right)^{-1 / 4}\left(n_{\beta} q^{2}+2|m-z|\right)^{-1 / 4}}{l_{\alpha} p^{2}+2 l(p, q)+l_{\beta} q^{2}+2|m-z|},
\end{aligned}
$$

where

$$
\mathrm{d}_{1}=\left(2 \pi^{2}\right)^{-1} l_{1}^{3 / 4} l_{2}^{3 / 4} .
$$

Here the equivalence is performed by the unitary dilation

$$
\mathbf{Y}=\operatorname{diag}\left\{Y_{1}, Y_{2},\right\}: L_{2}^{(2)}\left(U_{\delta}(0)\right) \rightarrow L_{2}^{(2)}\left(\hat{U}_{\delta}(0)\right), \quad\left(Y_{\alpha} f\right)(p)=f\left(U^{-\frac{1}{2}} p\right) .
$$

The operator $T_{0}(\delta ;|m-z|)$ is unitary equivalent to the integral operator $T_{1}(r)$ acting in $L_{2}^{(2)}\left(U_{r}(0)\right)$, where $r=|m-z|^{-\frac{1}{2}}$ and $U_{r}(0)=\left\{p \in \mathbb{R}^{3}:|p|<r\right\}$, with the kernel

$$
\begin{aligned}
& T_{\alpha \alpha}^{(1)}(r ; p, q)=0, \\
& T_{\alpha \beta}^{(1)}(r ; p, q)=\mathrm{d}_{1} \frac{\left(n_{\alpha} p^{2}+2\right)^{-1 / 4}\left(n_{\beta} q^{2}+2\right)^{-1 / 4}}{l_{\alpha} p^{2}+2 l(p, q)+l_{\beta} q^{2}+2} .
\end{aligned}
$$


The equivalence is performed by the unitary dilation

$$
\mathbf{B}_{r}=\operatorname{diag}\left\{B_{r}, B_{r}\right\}: L_{2}^{(2)}\left(U_{\delta}(0)\right) \rightarrow L_{2}^{(2)}\left(U_{r}(0)\right), \quad\left(B_{r} f\right)(p)=\left(\frac{r}{\delta}\right)^{-3 / 2} f\left(\frac{\delta}{r} p\right) .
$$

Further, we may replace

$$
\left(n_{\alpha} p^{2}+2\right)^{-1 / 4},\left(n_{\beta} q^{2}+2\right)^{-1 / 4} \quad \text { and } \quad l_{\alpha} p^{2}+2 l(p, q)+l_{\beta} q^{2}+2
$$

by

$$
\left(n_{\alpha} p^{2}\right)^{-1 / 4}\left(1-\chi_{1}(p)\right),\left(n_{\beta} q^{2}\right)^{-1 / 4}\left(1-\chi_{1}(q)\right) \quad \text { and } \quad l_{\alpha} p^{2}+2 l(p, q)+l_{\beta} q^{2},
$$

respectively, since the error will be a Hilbert-Schmidt operator continuous up to $z=m$, where $\chi_{1}(\cdot)$ is a characteristic function of the ball $U_{1}(0)$. Then we get the integral operator $T_{2}(r)$ in $L_{2}^{(2)}\left(U_{r}(0) \backslash U_{1}(0)\right)$ with the kernel

$$
\begin{aligned}
& T_{\alpha \alpha}^{(2)}(r ; p, q)=0, \\
& T_{\alpha \beta}^{(2)}(r ; p, q)=\frac{\mathrm{d}_{1}}{\left(n_{1} n_{2}\right)^{\frac{1}{4}}} \frac{|p|^{-1 / 2}|q|^{-1 / 2}}{l_{\alpha} p^{2}+2 l(p, q)+l_{\beta} q^{2}} .
\end{aligned}
$$

By the dilation

$$
\mathbf{M}=\operatorname{diag}\{M, M\}: L_{2}^{(2)}\left(U_{r}(0) \backslash U_{1}(0)\right) \rightarrow L_{2}\left((0, \mathbf{r}), \sigma^{(2)}\right), \mathbf{r}=1 / 2|\log | m-z||,
$$

where $(M f)(x, w)=e^{3 x / 2} f\left(e^{x} w\right), \quad x \in(0, \mathbf{r}), w \in \mathbb{S}^{2}$, one sees that the operator $T_{2}(r)$ is unitarily equivalent to the integral operator $\mathbf{S}_{\mathbf{r}}$ with entries 6.3 .

The difference of the operators $\mathbf{S}_{\mathbf{r}}$ and $T(\delta ;|m-z|)$ is compact (up to unitarily equivalence) and hence, taking into account that $\mathbf{r}=1 / 2|\log | m-z \mid$, we obtain

$$
\lim _{|z-m| \rightarrow 0}|\log | z-\left.m\right|^{-1} n(\mu, T(\delta ;|z-m|))=U(\mu), \quad \mu>0 .
$$

Theorem 6.4 is proved.

Proof of part $(i i)$ of Theorem 2.10 Let the conditions in part (ii) of Theorem 2.10 be fulfilled. Then the equality

$$
\lim _{|z-m| \rightarrow 0}|\log | z-m||^{-1} n(1, T(z))=U(1)
$$

follows from Lemmas 6.1 6.2 and Theorem 6.4 Taking into account the equality 6.4 and using Lemma 4.4 we complete the proof of part (ii) of Theorem 2.10

Acknowledgement The authors would like to thank Prof. R. A. Minlos for helpful discussions about the results of this paper. This work was supported by the DFG 436 USB 113/4 Project and the Fundamental Science Foundation of Uzbekistan. S. N. Lakaev and Z. I. Muminov gratefully acknowledge the hospitality of the Institute of Applied Mathematics and of the IZKS of the University of Bonn.

\section{REFERENCES}

[1] S. Albeverio, R. HøEGH-Krohn, and T. T. WU: A class of exactly solvable three-body quantum mechanical problems and universal low energy behavior. Phys. Lett. A 83 (1971), 105-109.

[2] S. Albeverio, S. N. LaKaev, K. A. MaKarov: The Efimov Effect and an Extended Szegö-Kac Limit Theorem. Letters in Math. Phys, V. 43 (1998),pp. 73-85.

[3] S. Albeverio, S. N. Lakaev, Z. I. Muminov: Schrödinger operators on lattices. The Efimov effect and discrete spectrum asymptotics. Ann. Henri Poincaré. $5743-772$ (2004).

[4] S. Albeverio, S. N. Lakaev, Z. I. Muminov: On the structure of the essential spectrum for the threeparticle Schrödinger operators on lattices. to appear in Math. Nach.(2006). 
[5] Albeverio S., Lakaev S. N. and Muminov Z. I. : The threshold effects for a family of Friedrichs models under rank one perturbations. arXiv:math.SP/0604277 v1 12 Apr 2006

[6] R. D. Amado and J. V. Noble: Efimov effect; A new pathology of three-particle Systems. II. Phys. Lett. B.35. No.1, 25-27, (1971); Phys. Lett. D.5. No.8, (1972), 1992-2002.

[7] G. F. Dell' Antonio, R. Figari, A. Teta: Hamiltonians for systems of $N$ particles interacting through point interactions. Ann. Inst. H. Poincaré Phys. Théor. 60 (1994), no. 3, 253-290.

[8] V. EFIMOV: Energy levels of three resonantly interacting particles. Nucl. Phys. A 210 (1973), 157-158.

[9] G. M. Graf And D. SchenKer: 2-magnon scattering in the Heisenberg model. Ann. Inst. H. Poincaré Phys. Théor. 67 91-107 (1997).

[10] FARia da Veiga P. A., IORIATti L., AND O'CARroll M.: Energy-momentum spectrum of some twoparticle lattice Schrödinger Hamiltonians. Phys. Rev. E (3) 66, 016130, 9 pp. (2002).

[11] Friedrichs K. O.: On the perturbation of continuous spectra. Communications on Appl. Math. 1(1948), 361-406.

[12] L. D. FAdDEEV: On a model of Friedrichs in the theory of perturbations of the continuous spectrum. (Russian) Trudy Mat. Inst. Steklov 73 (1964), 292-313.

[13] L. D. FAdDEev and S. P. MERKuRIEv: Quantum scattering theory for several particle systems. Kluwer Academic Publishers, 1993.

[14] S. N. LAKAEV: On an infinite number of three-particle bound states of a system of quantum lattice particles. Theor. and Math. Phys. 89 (1991), No.1, 1079-1086.

[15] S. N. LAKAEv: The Efimov's Effect of a system of Three Identical Quantum lattice Particles. Funkcionalnii analiz i ego priloj., 27 (1993), No. 3, pp. 15-28, translation in Funct. Anal.Appl.

[16] V. A. Malishev And R. A. Minlos: Linear infinite-particle operators. Translations of Mathematical Monographs, 143. American Mathematical Society, Providence, RI, 1995.

[17] D. C. MatTis: The few-body problem on lattice. Rev. Modern Phys. 58 (1986), No. 2, 361-379

[18] A. I. MogILNER: Hamiltonians of solid state physics at few-particle discrete Schrödinger operators: problems and results. Advances in Sov. Math., 5 (1991), 139-194

[19] YU. N. OvchinNiKov AND I. M. SigAL: Number of bound states of three-particle systems and Efimov's effect. Ann. Physics, 123 (1989), 274-295

[20] M. ReED and B. SimON: Methods of modern mathematical physics. III: Scattering theory. Academic Press, N.Y., 1979.

[21] M. ReED and B. Simon: Methods of modern mathematical physics. IV: Analysis of Operators. Academic Press, N.Y., 1979.

[22] A. V. Sobolev: The Efimov effect. Discrete spectrum asymptotics. Commun. Math. Phys. 156 (1993), $127-168$.

[23] H. TAmura: The Efimov effect of three-body Schrödinger operator. J. Funct. Anal. 95 (1991), 433-459.

[24] H. TAMURA: Asymptotics for the number of negative eigenvalues of three-body Schrödinger operators with Efimov effect. Spectral and scattering theory and applications. 23, 1994, 311-322, Adv. Stud. Pure Math., Math. Soc. Japan, Tokyo.

[25] D. R. YAFAEV: On the theory of the discrete spectrum of the three-particle Schrödinger operator. Math. USSR-Sb. 23 (1974), 535-559.

[26] D. R. YAFAEV: Mathematical scattering theory; general theory. Translations of mathematical Monographs, 105. American Mathematical society, Providence 1992.

[27] D. R. YAfAEv: Scattering theory: Some old and new problems. Lecture Notes in Mathematics, 1735. Springer-Verlag, Berlin, 2000, 169 pp.

${ }^{1}$ Institut FÜr Angewandte Mathematik, Universität Bonn, Wegelerstr. 6, D-53115 BonN (GERMANY)

2 SFB 611, BONN, BiBoS, BIELEFELD - BONN;

3 CERFIM, LOCARNO AND ACC.ARCH,USI (SwITZERLAND)E-MAIL: ALBEVERIO@UNI.BONN.DE

${ }^{4}$ Samarkand State University, University BouleVard 15, 703004, Samarkand (UzBeKISTAN) E-MAIL: SLAKAEV@MAIL.RU

${ }^{5}$ SAMARKAND DIVISION OF ACADEMY OF SCIENCES OF UZBEKISTAN (UZBEKISTAN) E-MAIL: ZIMUMINOV@MAIL.RU 